\title{
Caries risk assessment models in caries prediction
}

\author{
Amila Zukanović \\ Department of Preventive and \\ Pediatric Dentistry, Faculty of \\ Dentistry University of Sarajevo \\ Sarajevo, Bosnia and Herzegovina \\ Corresponding author: \\ Amila Zukanović \\ Department of Preventive and \\ Pediatric Dentistry, Faculty of \\ Dentistry, University of Sarajevo \\ Bolnička 4a \\ 71000 Sarajevo \\ Bosnia and Herzegovina \\ amila.zukanovic@hotmail.com \\ Tel.: + 38761772161 \\ Fax.: + 38733211941
}

Received: 13 March 2013

Accepted: 26 April 2013

Copyright (C) 2013 by

Academy of Sciences and Arts of Bosnia and Herzegovina. E-mail for permission to publish: amabih@anubih.ba

\begin{abstract}
Objective. The aim of this research was to assess the efficiency of different multifactor models in caries prediction. Material and methods. Data from the questionnaire and objective examination of 109 examinees was entered into the Cariogram, Previser and Caries-Risk Assessment Tool (CAT) multifactor risk assessment models. Caries risk was assessed with the help of all three models for each patient, classifying them as low, medium or high-risk patients. The development of new caries lesions over a period of three years [Decay Missing Filled Tooth $(\mathrm{DMFT})$ increment $=$ difference between Decay Missing Filled Tooth Surface (DMFTS) index at baseline and follow up], provided for examination of the predictive capacity concerning different multifactor models. Results. The data gathered showed that different multifactor risk assessment models give significantly different results (Friedman test: Chi square $=100.073, p=0.000$ ). Cariogram is the model which identified the majority of examinees as medium risk patients (70\%). The other two models were more radical in risk assessment, giving more unfavorable risk -profiles for patients. In only $12 \%$ of the patients did the three multifactor models assess the risk in the same way. Previser and CAT gave the same results in $63 \%$ of cases - the Wilcoxon test showed that there is no statistically significant difference in caries risk assessment between these two models $(Z=-1.805$, $\mathrm{p}=0.071$ ). Conclusions. Evaluation of three different multifactor caries risk assessment models (Cariogram, PreViser and CAT) showed that only the Cariogram can successfully predict new caries development in 12-year-old Bosnian children.
\end{abstract}

Key words: Caries risk assessment, Multifactorial model, Prediction.

\section{Introduction}

Caries risk is defined as the probability of an individual developing at least a certain number of caries lesions during specific period of time (1). Risk assessment is important step in decision-making and treatment planning. Routine preventive measures and recommendations are very effective within the general population but they do not target patients that are at greater than average risk (2). Caries risk assessment is a very complex issue, due to its multicausal etiology, the numerous and complex relations and interactions between caries risk factors, and the dependency of each factor on dose, frequency and duration. Many models for caries risk assessment (CRA) had been suggested, including different numbers and combinations of caries risk factors as caries predictors, different modes of evaluation and interpretation of risk assessment results. Models that contain only one or two 
risk factors, cannot predict future caries development successfully $(3,4,5)$. In assessing the risk, more complex models, that operate with several risk factors, should be used. Nowadays, there are few risk models based on a multifactor concept $(6,7,8)$. Caries risk models should also evaluate risk factors together and simultaneously, so some of the recent CRA models are based on information technology (9).

Most research has analyzed the role and power of single or mulitple risk factors as predictors of future caries development, but very little research has evaluated the success of multifactor models suggested for caries risk assessment, as shown in Siyama and al.s review of literature (10). Accuracy of prediction models must be determined in longitudinal studies, but most research has a crosssectional study design (11).

The aim of this study was to assess the efficiency of three different, multifactor caries risk assessment models [Cariogram, Previser and Caries-Risk Assessment Tool (CAT)], by comparing caries risk assessment with the actual caries increment in 12-year-old children, over a 3 -year period.

\section{Subjects and methods}

\section{Subjects}

The study population consisted of 109 schoolchildren who were 12-years old at the start of the study. All the children live in Sarajevo, the capital of Bosnia and Herzegovina (the total population of Sarajevo is nearly 450,000 inhabitants). Three elementary schools were selected - a private school where parents pay full school fees, a private school where underprivileged children have free education, and one state-run school from the Novo Sarajevo municipality. 40 pupils were selected randomly from each school. Eleven children did not participate in the study ( 5 children chose not to participate in the study, 6 children did not come to examinations). This resulted in a study population of 109 children at baseline, 60 boys and 49 girls. The follow-up study was conducted 3 years later. The children were reexamined by the same examiner, using the same procedure as that practiced at baseline. The follow-up sample included 70 children (64.2\% of the initial study population), 38 boys and 32 girls. School principals, teachers and children were given written information about the study. The parents signed informed consent forms for participation in the study. The Ethical Committee of the University of Sarajevo, Bosnia and Herzegovina approved the study.

In Bosnia and Herzegovina, all children up to age of 18 have free access to regular dental care at public dental clinics. During the period of three years, the participants in the study received regular dental care.

\section{Study design}

At baseline, the study consisted of the following steps: the questionnaire and an interview, clinical examination, saliva sampling, and caries risk assessment using CRA models. Children completed questionnaires in the school classrooms, prior to examinations. The questionnaire contained questions about: general data, questions about socioeconomic background, diet, fluoride program, tooth brushing habits, medical history and dental care. Clinical examinations consisted of: scoring of oral hygiene using the Silness - Löe plaque index, determination of dental status [Decay Missing Filling Tooth (DMFT) and Decay Missing Filled Tooth Surface (DMFS) indexes], gingival check-up (changes in colour, shape, structure and consistency of gingiva). Clinical examination was carried out by the same examiner following WHO criteria (12). After the questionnaire, interview and clinical examination, saliva sampling was performed for each child. Saliva sampling consisted of: 
measurements of mutans streptococci in the saliva using a Dentocult SM Strip mutans test, measurements of lactobacilli in the saliva using a Dentocult ${ }^{\circ}$ LB test, measurements of saliva buffer capacity using a Dentobuff Strip test, measurement of the saliva secretion rate (paraffin stimulated saliva for 5 minutes). All tests were from Orion Diagnostica, Espoo, Finland.

Caries risk assessment, using different multifactor caries risk assessment models, consisted of the following steps. All data gained from the questionnaire and interview, clinical examination and saliva sampling are actually caries related factors/parameters needed for creation of risk profiles, based on three different caries risk assessment models. The various parameters are given a score according to predetermined scales for each CRA model (Table 1). Different combinations of scored factors/parameters are entered into each of the CRA models - Cariogram, PreViser and CAT model and caries risk assessment is performed for each examinee.

Table 1 Caries-related factors and scores used for Cariogram, PreViser and CAT models

\begin{tabular}{|c|c|}
\hline \multicolumn{2}{|c|}{ Diet, contents (based on the lactobacillus test counts) } \\
\hline Score 0 & Very low fermentable carbohydrate intake ( $<1.000 \mathrm{CFU} / \mathrm{ml})$ \\
\hline Score 1 & Low fermentable carbohydrate intake (10.000 CFU/ml) \\
\hline Score 2 & Moderate fermentable carbohydrate intake(100.000 CFU/ml) \\
\hline Score 3 & High fermentable carbohydrate intake (>1.000.000 CFU/ml) \\
\hline \multicolumn{2}{|c|}{ Diet, frequency (estimation of the number of meals and snacs per day) } \\
\hline Score 0 & Maximum 3 meals per day \\
\hline Score 1 & 4-5 meals per day \\
\hline Score 2 & 6-7 meals per day \\
\hline Score 3 & $>7$ meals per day \\
\hline \multicolumn{2}{|c|}{ Oral hygiene (based on the plaque amount) } \\
\hline Score 0 & Very good oral hygiene (Plaque index $<0.4$ ) \\
\hline Score 1 & Good oral hygiene (Plaque index $=0.4-1.0$ ) \\
\hline Score 2 & Poor oral hygiene (Plaque index $=1.1-2.0$ ) \\
\hline Score 3 & Very poor oral hygiene (Plaque index $>2.0$ ) \\
\hline \multicolumn{2}{|c|}{ Mutans streptococcus (estimation of the level of mutans streptococcus in saliva) } \\
\hline Score 0 & Very low or zero amounts $(<20.000 \mathrm{CFU} / \mathrm{ml})$ \\
\hline Score 1 & Low level $(20.000-100.000$ CFU/ml) \\
\hline Score 2 & High amounts (100.000 - 1.000.000 CFU/ml) \\
\hline Score 3 & Very high amounts (>1.000.000 CFU/ml) \\
\hline \multicolumn{2}{|c|}{ Fluoride program (estimation of the extent of fluoride available in the oral cavity) } \\
\hline Score 0 & Maximum fluoride program \\
\hline Score 1 & Fluoride supplements, irregularly \\
\hline Score 2 & Fluoride toothpaste only \\
\hline Score 3 & No fluoride \\
\hline \multicolumn{2}{|c|}{ Saliva secretion (estimation of the flow rate of stimulated saliva) } \\
\hline Score 0 & Normal saliva secretion ( $\geq 0.7 \mathrm{ml} / \mathrm{min}$ ) \\
\hline Score 1 & Low saliva secretion $(0.3-0.7 \mathrm{ml} / \mathrm{min})$ \\
\hline Score 2 & Very low saliva secretion $(\leq 0.3 \mathrm{ml} / \mathrm{min})$ \\
\hline \multicolumn{2}{|c|}{ Saliva buffering capacity (estimation of saliva to buffer acids) } \\
\hline Score 0 & Adequate buffer capacity $(\mathrm{pH} \geq 6.0)$ \\
\hline Score 1 & Reduced buffer capacity (pH 4.5-5.5) \\
\hline Score 2 & Low buffer capacity $(\mathrm{pH} \leq 4.0)$ \\
\hline
\end{tabular}


Continuation of Table 1 Caries-related factors and scores used for Cariogram, PreViser and CAT models

\begin{tabular}{|c|c|}
\hline \multicolumn{2}{|c|}{ Socioeconomic status (estimation of socioeconomic status of child/child's parent) } \\
\hline Score 0 & High \\
\hline Score 1 & Mid-level \\
\hline Score 2 & Low \\
\hline \multicolumn{2}{|c|}{ Visit to dentist } \\
\hline Score 0 & Regular use of dental care \\
\hline Score 1 & Irregular use of dental care \\
\hline Score 2 & No usual source of dental care \\
\hline \multicolumn{2}{|c|}{ Child has decay? } \\
\hline Score 0 & No \\
\hline Score 1 & Yes \\
\hline \multicolumn{2}{|c|}{ Times per day that child teeth are brushed } \\
\hline Score 0 & 2-3 times per day \\
\hline Score 1 & 1 per day \\
\hline Score 2 & $<1$ times per day \\
\hline \multicolumn{2}{|c|}{ Gingivitis (red, puffy gums) } \\
\hline Score 0 & Absent \\
\hline Score 1 & Present \\
\hline \multicolumn{2}{|l|}{ Dental floss } \\
\hline Score 0 & Regular use of dental floss \\
\hline Score 1 & Irregular use of dental floss \\
\hline \multicolumn{2}{|c|}{ Time lapsed since child's last cavity } \\
\hline Score 0 & 24-35 months \\
\hline Score 1 & $12-23$ months \\
\hline Score 2 & $<12$ months \\
\hline \multicolumn{2}{|l|}{ Sealants } \\
\hline Score 0 & Yes \\
\hline Score 1 & No \\
\hline \multicolumn{2}{|c|}{ Medical history (general diseases or conditions associated to dental caries) } \\
\hline Score 0 & No disease, healthy \\
\hline Score 1 & A general disease that can influence the caries process to a mild degree \\
\hline Score 2 & A general disease that can influence the caries process to a high degree \\
\hline \multicolumn{2}{|c|}{ Caries experience (DMFT index at baseline) } \\
\hline Score 0 & $\mathrm{DMFT}=0$ \\
\hline Score 1 & $\mathrm{DMFT}=1$ \\
\hline Score 2 & $\mathrm{DMFT}=2$ \\
\hline \multicolumn{2}{|c|}{ Child wears braces or orthodontic/oral appliances? } \\
\hline Score 0 & No \\
\hline Score 1 & Yes \\
\hline \multicolumn{2}{|c|}{ Child has decay? } \\
\hline Score 0 & Yes \\
\hline Score 1 & No \\
\hline
\end{tabular}

CAT=Caries-Risk Assessment Tool; CFU=Colony Forming Units; DMFT=Decay Missing Filling Tooth.

\section{Cariogram}

Cariogram is an interactive computer program which assesses risk of new caries le- sion development. It presents the caries risk profile of an individual graphically, simultaneously taking into account the interac- 
tion of different causative factors of caries. It creates an individual future „risk scenario“, based on the given scores and interactions of 9 factors/parameters of direct relevance to caries, entered in the Cardiogram. The factors are: caries experience, related diseases, diet content and frequency, plaque amount, mutans streptococci level, fluoride program, saliva secretion and buffering capacity ( $\mathrm{Ta}$ ble 1). The Cariogram contains many „if“ conditions - it can operate with 5 million combinations of caries related factors (13). According to the weighted formula, after all data of relevance for caries are collected from the individuals, scored and entered in the Cariogram, the program presents a pie diagram with the following sectors: bacteria, diet, susceptibility and circumstances. The caries risk is express in the sector „chance of avoiding caries". When the chance of avoiding caries is high, the caries risk is small and vice versa. The chance varies on a scale from 0 to $100 \%$ - chance from 0 to $20 \%$ means that the individual has high caries risk, from 21 to $80 \%$ medium risk and from $81-100 \%$ low risk for future caries development.

\section{PreViser - oral health risk assessment software}

PreViser, Inc. is an evidence-based online risk prediction system that uses software technology to predict common oral diseases, specifically periodontitis, caries and oral cancer. PreViser is the part of OHIS ${ }^{\mathrm{TM}}$ (Oral Health Information System) and contains three different parts: a Periodontal Assessment Tool (PAT), a Caries Risk Tool and an Oral Cancer Risk Tool. The "Caries (tooth decay), Root, and Fracture Risk Assessment" input form for patient age 9-18, consists of questions about patient history and clinical data (general information about the patient, data about the tooth most recently exposed to saliva for the last 12 months and months that patient has been caries free, data about oral hygiene, diet content and frequency, fluoride program and orthodontic appliances). PreViser calculates risk online, in a central software unit, and expresses it as low/moderate/high risk. High risk means that the patient is very likely to have a cavity within the next 3 years.

\section{Caries-Risk Assessment Tool}

The Caries-Risk Assessment Tool (CAT) is the tool for risk assessment of infants, children and adolescents, supported by the American Academy of Pediatric Dentistry. This tool is based on a set of physical, environmental and general health factors. Factors evaluated in the CAT, determined by interviewing the parent/primary caregiver are: exposure to fluoride, dietary and oral hygiene habits, socio-economical status, dental care, medical conditions that impact motor coordination/cooperation or impair saliva, orthodontic/oral appliances, the time lapsed since child's last cavity. Clinical evaluation connotes visible plaque, gingivitis, presence of enamel demineralization, enamel defects, deep pits and fissures, and previous caries experience. Radiography and saliva tests (levels of mutans streptococci and lactobacilli) are not essential for using CAT (supplemental professional assessment). Depending on the score/value given to the parameters, individual overall caries risk assessment is based on the highest level of any aforesaid risk indicator (low/medium/high risk).

The caries increment was estimated after 3 years. At follow up, all the children were examined by the same examiner. Following the same diagnostic criteria and procedure as done at baseline, the DMFT and DMFS index was computed for each examinee.

\section{Statistical methods}

Results are shown using descriptive statistics (frequency distributions, mean $\pm \mathrm{SD}$ ) and presented in tables and figures. The Kappa 
coefficient was used to evaluate the extent of agreement between risk profiles assessed by two different CRA models. To test the differences in caries risks, assessed with three different CRA models (Cariogram, PreViser and CAT), a non-parametric Friedman test was used. The Wicoxon signed rank test was used to test the differences between two risk profiles. The level of significance was set at $\mathrm{p}<0.05$. Logistic regression analyses were carried out to test CRA models to predict the development of new caries lesions. The response variable was DMFS increment (difference between DMFS at baseline and follow-up), presented as no new caries/new caries over a period of 3 years. For statistical analyses, SPSS 15.0 (SPSS, Inc, Chicago, Il) version 9 software was used.

\section{Results}

The figure presents caries risk assessment results at baseline for 109 examinees with three different multifactor models. According to the Cariogram, $70.9 \%$ of children were in the medium risk group and only $7.3 \%$ in the high risk group. According to
PreViser and CAT the majority of children were in the high risk group.

Risk assessed with Cariogram and Previser was identical in only $30.02 \%$ of individuals. In the group of $30.02 \%$ identical risk profiles, $10.1 \%$ of the examinees showed assessed risk as low, $12.8 \%$ as medium and $7.3 \%$ as high. Cariogram and CAT created identical risk profiles in only $19.2 \%$ cases (6.4\% low, $5.5 \%$ medium and $7.3 \%$ high caries risk). Agreement of risk profiles between Previser and CAT was found in $62.4 \%$ examinees $(3.7 \%$ low risk, $1.8 \%$ medium risk and $56.9 \%$ high risk).

To evaluate the extent of agreement between risk assessed by different models, the Kappa coefficient was used. There was poor agreement between Cariogram and PreViser (kappa coefficient $=0.139, \mathrm{p}=0.000$ ), Cariogram and the CAT model (Kappa coefficient $=0.053, \mathrm{p}=0.049)$ and between PreViser and CAT (Kappa coefficient $=0.103$, $\mathrm{p}=0.143$ ).

To test the differences between caries risk profiles assessed with Cariogram, PreViser and CAT, the non-parametric Friedeman test was used. Mean ranks were: Cariogram 1.36,

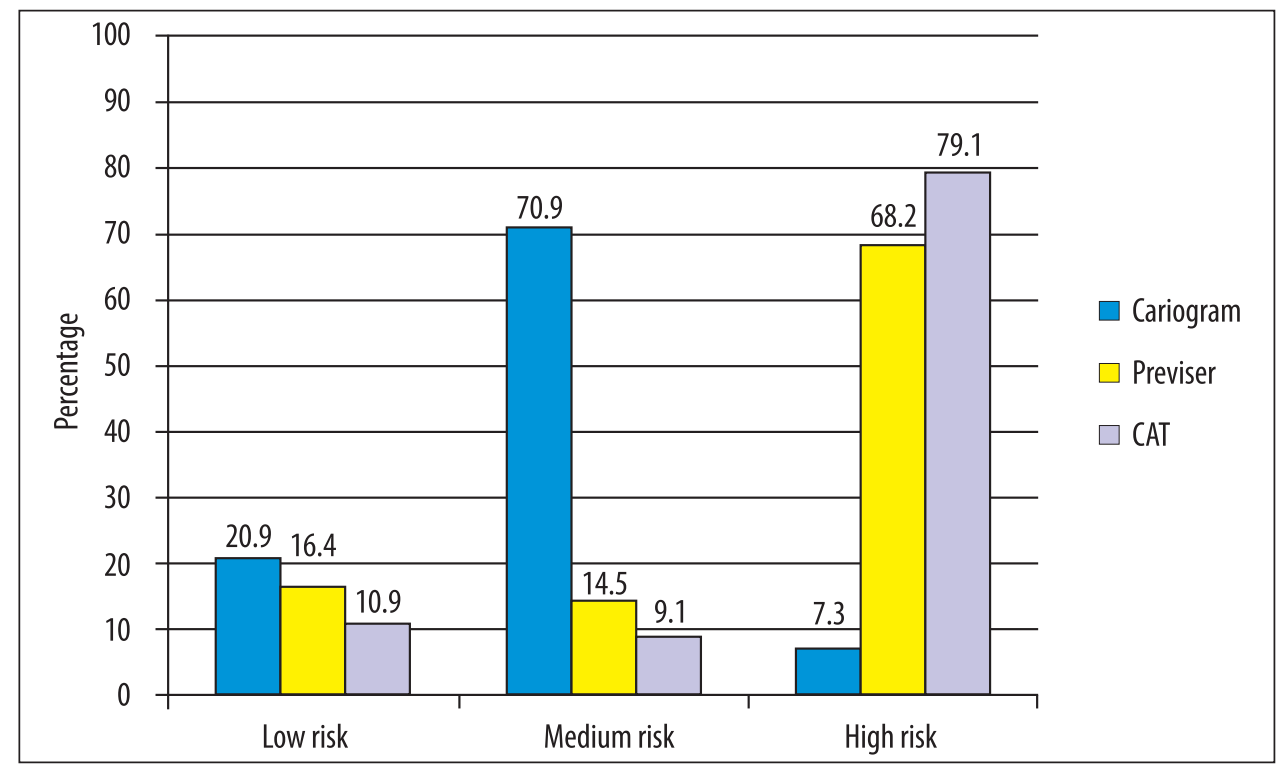

Figure 1 Caries risk assessment at baseline using Cariogram, Previser and CAT models. 
Table 2 Cross-tabulation between individual risk profiles assessed with Cariogram, PreViser and CAT

\begin{tabular}{|c|c|c|c|c|c|}
\hline & & PreViser & & & \\
\hline Cross-tabul & & Low risk & Medium risk & High risk & Total \\
\hline & Low risk & $11(10.1)$ & $2(1.8)$ & $10(9.2)$ & $23(21.1$ \\
\hline . & Medium risk & $7(6.4)$ & $14(12.8)$ & $57(52.3)$ & 78 (71.6) \\
\hline cantogram & High risk & $0(0)$ & $0(0)$ & $8(7.3)$ & $8(7.3)$ \\
\hline & Total & $18(16.5)$ & $16(14.7)$ & $75(68.8)$ & $109(100)$ \\
\hline & & CAT & & & \\
\hline & Low risk & $7(6.4)$ & $4(3.7)$ & $12(11.0)$ & $23(21.1)$ \\
\hline$C_{0} \cdot$ & Medium risk & $5(4.6)$ & $6(5.5)$ & $67(61.5)$ & 78 (71.6) \\
\hline Callogiam & High risk & $0(0.0)$ & $0(0.0)$ & $8(7.3)$ & $8(7.3)$ \\
\hline & Total & $12(11.0)$ & $10(9.2)$ & 87 (79.8) & 109 (100.0) \\
\hline & & CAT & & & \\
\hline & Low risk & $4(3.7)$ & $1(0.9)$ & $13(11.9)$ & $18(16.5)$ \\
\hline$\Omega_{0}$ & Medium risk & $2(1.8)$ & $2(1.8)$ & $12(11.0)$ & $16(14.7)$ \\
\hline Previser & High risk & $6(5.5)$ & $7(6.4)$ & $62(56.9)$ & $75(68.8)$ \\
\hline & Total & $12(11.0)$ & $10(9.2)$ & 87 (79.8) & 109 (100.0) \\
\hline
\end{tabular}

CAT= Caries-Risk Assessment Tool.

Previser 2.23, CAT 2.41. The test showed that there are significant differences between risks assessed with three different CRA models (Chi square $=100.073, \mathrm{p}=0.000)$.

The Wilcoxon signed rank test was performed to test the differences in risk profiles between the two related groups. The results showed that there are significant differences between caries risk assessed with the Cariogram and the PreViser $(\mathrm{Z}=-6.944, \mathrm{p}=0.000)$ models and between risk assessed with Cariogram and CAT $(\mathrm{Z}=-8.010, \mathrm{p}=0.000)$. There was no significant difference between individual risk assessed with PreViser and CAT $(\mathrm{Z}=-1.805, \mathrm{p}=0.071)$.

Table 3 presents the Cariogram, PreViser and CAT risk profiles (assessed as low/medium/high) related to DMFT and DMFS indexes means at baseline and follow-up and actual caries increment of DMFT and DMFS indexes over the period of three years.

Omnibus tests of model coefficients showed Chi - square equal to 6.172 on 2 degrees of freedom $(p=0.046)$, indicating that at least one of the covariates is signifi- cantly associated with caries increment over 3 years. Based on pseudo $R$ square values $($ Cox\&Snell R square $=0.084$, Nagelkerke $\mathrm{R}$ Square $=0.141$ ), it can be concluded that between 8.4 and 14.1 per cent of variability is explained by this set of variables. The Hosmer-Lemesh test (Chi square $=0.000$, $\mathrm{p}=1.000$ ) supports our prediction model (binary logistic model with forward LR method), as being worthwhile.

Table 4 presents the results of binary logistic regression for all three CRA models. The dependent variable was the DMFS increment (difference between DMFS at baseline and follow-up), presented as dichotomous variables (no new caries/new caries, coded $0 / 1$ ) over a period of 3 years. As independent variables, the value (low/medium/high risk) of risk profiles for each of the model (Cariogram, PreViser, CAT) was entered in the binary logistic model separately. Table shows that only the Cariogram can predict the development of new caries lesions in the future $(\mathrm{p}<0.05)$. The lowest risk group (Cariogram 0 ) was chosen as the 
Table 3 Caries risk assessment made by Cariogram, Previser and CAT and DMFT and DMF(S) increment over a period of 3 years

\begin{tabular}{|c|c|c|c|}
\hline \multirow{2}{*}{ Caries risk assessment } & \multicolumn{3}{|l|}{ Risk } \\
\hline & Low risk & Medium risk & High risk \\
\hline Individuals at baseline & \multicolumn{3}{|c|}{$n(\%)$} \\
\hline Cariogram & $23(21.10)$ & $78(71.56)$ & $8(7.34)$ \\
\hline Previser & $18(16.51)$ & $16(14.68)$ & $75(68.81)$ \\
\hline CAT & $12(11.01)$ & $10(9.17)$ & $87(79.82)$ \\
\hline Individuals at follow-up & \multicolumn{3}{|c|}{$n(\%)$} \\
\hline Cariogram & $13(18.57)$ & $52(74.29)$ & $5(7.14)$ \\
\hline Previser & $11(15.71)$ & $13(18.57)$ & $46(65.71)$ \\
\hline CAT & $8(11.43)$ & $5(7.14)$ & $57(81.43)$ \\
\hline DMFT at baseline & \multicolumn{3}{|c|}{$($ mean $\pm S D)$} \\
\hline Cariogram & $2.13 \pm 2.67$ & $5.50 \pm 3.12$ & $5.75 \pm 1.58$ \\
\hline Previser & $0.67 \pm 0.97$ & $3.69 \pm 2.12$ & $6.04 \pm 2.86$ \\
\hline CAT & $2.42 \pm 2.50$ & $4.10 \pm 2.42$ & $5.22 \pm 3.27$ \\
\hline DMFT at follow-up & \multicolumn{3}{|c|}{$($ mean $\pm S D)$} \\
\hline Cariogram & $4.15 \pm 3.31$ & $8.02 \pm 4.03$ & $8.00 \pm 3.08$ \\
\hline Previser & $2.91 \pm 2.43$ & $5.54 \pm 3.62$ & $8.85 \pm 3.58$ \\
\hline CAT & $4.75 \pm 3.58$ & $8.40 \pm 2.70$ & $7.56 \pm 4.17$ \\
\hline DMFT increment & \multicolumn{3}{|c|}{$($ mean $\pm S D)$} \\
\hline Cariogram & $1.77 \pm 1.88$ & $2.40 \pm 2.36$ & $1.80 \pm 1.79$ \\
\hline Previser & $2.18 \pm 2.32$ & $1.92 \pm 2.18$ & $2.35 \pm 2.27$ \\
\hline CAT & $2.38 \pm 1.92$ & $2.60 \pm 1.82$ & $2.19 \pm 2.33$ \\
\hline $\mathrm{DMF}(\mathrm{S})$ increment & \multicolumn{3}{|c|}{$($ mean $\pm S D)$} \\
\hline Cariogram & $2.54 \pm 2.44$ & $4.71 \pm 4.34$ & $5.00 \pm 7.07$ \\
\hline Previser & $2.82 \pm 3.19$ & $3.08 \pm 2.87$ & $5.04 \pm 4.75$ \\
\hline CAT & $3.13 \pm 2.53$ & $3.80 \pm 5.81$ & $4.54 \pm 4.41$ \\
\hline
\end{tabular}

CAT=Caries-Risk Assessment Tool; DMFT=Decay Missing Filling Tooth.

Table 4 Logistic regression model for Cariogram, Previser and CAT

\begin{tabular}{lllllllll}
\hline Covariates & B & S.E. & Wald & df & $p$ & Exp (B) & $95.0 \%$ Cl for Exp (B) \\
\hline Cariogram 0 & - & - & 6.08 & 2 & 0.048 & - & Lower & Upper \\
Cariogram 1 & 1.609 & 1.12 & 2.04 & 1 & 0.153 & 5.00 & 0.551 & 45.39 \\
Cariogram 2 & 2.442 & 1.01 & 5.83 & 1 & 0.016 & 11.50 & 1.58 & 83.38 \\
Constant & -0.405 & 0.913 & 0.197 & 1 & 0.657 & 0.667 & - & - \\
\hline
\end{tabular}

CAT $=$ Caries-Risk Assessment Tool.

reference value. Individuals with high caries risk (assessed with the Cariogram) have 11.5 times more chance of developing new caries lesions in the future, compared to low caries risk individuals (Odds ratio $=11.5, \mathrm{p}=0.016$, $\mathrm{CI}=1.58-83.3)$. 


\section{Discussion}

Caries risk assessment (CRA) is an important step in dental treatment based on the concept of minimally invasive therapy, where therapeutic and prophylactic measures are planned, based on the results of caries risk assessment. The use of numerous and non-standardized CRA protocols can lead to bias in caries risk assessment. The Moss study showed that equable criteria are essential for determination and assessment of high-risk patients (14), and these are provided by applying standardized multifactor CRA models. There is no recommendation for dental practitioners in Bosnia and Herzegovina as to which protocol or CRA model to use in caries prediction. Bader's research showed that even if a certain protocol for CRA was recommended, it remained unclear how it was used in dental practice (15) and even more, what was the reason for using a certain type of CRA protocol (16).

CRA models vary, from simple ones that operate with only one caries risk factor to complex, multifactor models that include more sophisticated methods, like microbial tests, saliva analyses and so on. Models that include only one caries - related factor or a combination of two risk factors, cannot predict the risk of future caries development successfully $(3,4,5)$. In our study, we used three different, standardized, multifactor CRA models (Cariogram, PreViser and CAT) to assess the risk in 12 year old children. The caries risk was categorized as low, medium or high, using all three models for each individual. The results showed that very often, CRA models assessed the risk differently - with one model, the child was assessed as a low risk but with another model as a medium, or high-risk patient. PreViser and CAT were more radical in CRA, giving more unfavorable risk profiles, compared to the Cariogram. According to the CAT model, almost $80 \%$ of children were in the high-risk group. The reason for this is probably the way of categorization of risk groups, where individual overall caries risk assessment is based on the highest level of any aforesaid risk indicator (low/medium/high risk). That way, the same importance was given to each of the caries risk factors included in the CAT model. What this means is that a patient with beneficial (categorized as low risk) values/scores of all factors and only one unfavorable factor (categorized as high risk) will be categorized as a "high risk patient". The PreViser model gave more favorable overall risk profiles for examinees than the CAT model, where about $70 \%$ children were in the high-risk group. The Cariogram model assessed less than $10 \%$ children as high risk. Its categorization into risk groups is more moderate compared with the other two models. All three models assess the risk identically for only $12 \%$ of examinees. The greatest agreement in results was shown by PreViser and CAT (63\% of cases), where the majority of children were in the high-risk group (57\%).

There is not much scientific evidence of the efficiency of multifactor risk models. Evaluation of the Cariogram model through longitudinal research (examinees being school children aged 10-11 and older examinees aged $55,65,75$ ) has showed that it can predict caries efficiently, better than any other model that includes single risk factors $(13,17,18)$. Based on the results of Hänsel-Petersson et al. from 2002 and 2003, Brathall D. concluded that the Cariogram is capable of sorting out individuals into riskgroups that have an actual chance of developing new caries lesions in the future (19). On the other hand, Holgerson et al. showed that a modified Cariogram applied to preschool children was not particularly useful in identifying high caries risk patients in a low-caries community (20). In the study of Utreja et al., conducted to evaluate the accuracy of the Cariogram in predicting the oc- 
currence of caries in first permanent molars in 30 children aged 8 , the results revealed that the Cariogram had a diagnostic accuracy of $63.33 \%$, thus emphasizing the need for better prediction models (21). In our study, the Cariogram was used on the basis of the original manual, without any modification of the program or risk factors entered into the Cariogram (22). The study results showed that the Cariogram can predict risk well in our examinees. Up until now, there has only been research proving PreViser efficacy in risk prediction, and that was only for the PAT module $(23,24,25,26)$. There has been no research to evaluate PreViser efficiency in caries risk assessment. The Caries risk Assessment Tool (CAT) helps in identification of caries risk factors, identification of the high-risk children and better caries prediction (27). Based on our results, PreViser and CAT cannot predict caries successfully in our examinees.

The analyses of the advisability and adequacy of risk factors entered into a certain multifactor risk model, the mode of registration and scoring of risk factors, are beyond the scope of this research. However, it should be noticed that there is still no international consensus about which factors in risk assessment should be considered and how to evaluate them (28). Zero et al. evaluated predictive power of multifactor CRA models in patients of various age. They showed that the predictive powers of different CRA models depend on the characteristics of the population for which the models are designed, and that any combination used for CRA is not constantly good when applied to different groups of examinees (e.g. different age groups) (29). Powel concludes that the CRA model must be adjusted to the patient's age as well as to caries prevalence inside the population (30). Each specific target population group (preschool children, teenagers, adults, and older patients) should have their own set of caries-risk variables to be considered during CRA. Taking all of the above into consideration, it should be noted that the Cariogram, the PreViser and the CAT were created and developed for populations of relatively low caries prevalence (USA, Sweden). It is somewhat expected that these models would not show equal efficiency when applied to 12-year-old Bosnian children (within a population of high caries prevalence and a completely different model of dental care compared to Sweden and the USA) (31). For these reasons, before we reject the models that did not show good efficiency in caries prediction (PreViser and CAT), the same models should be tested on a larger number of examinees from various populations and different age groups.

\section{Conclusion}

Within all the limitations of this study, it can be concluded that our results suggest that of the three different multifactor CRA models (Cariogram, PreViser and CAT) only the Cariogram can successfully predict new caries development in Bosnian 12-year-old children (a high risk community).

Acknowledgements: We are thankful to OrionDiagnostica Finland, the PreViser Corporation, the Sarajevo Canton Ministry of Education and Science, the Federal Ministry of Education and Science of Bosnia and Herzegovina and Department of Cariology of Faculty of Dentistry, Malmo University.

Conflict of interest: The author declares that she has no conflict of interest.

\section{References}

1. Kalwitzki M, Weiger R, Axmann - Krcmar D, Rosendahl R. Caries risk analysis: considering caries as an individual time - dependent process. Int J Paediatr Dent. 2002;12(2):132-42.

2. Berg JH. Dental caries detection and risk management by risk assessment. Journal Compilation. 2007;1(7):49-55.

3. Bratthall D, Ericsson D. Test for assessment of caries risk. In: Thylstrup A, Fejerskov O, editors. 
Textbook of Clinical cariology. 2nd ed. Copenhagen: Munksgaard; 1994. p. 333-53.

4. Reich E, Lussi A, Newbrun E. Caries - risk assessment. Int Dent J. 1999;49:15-26.

5. Steiner M, Helfenstein U, Marthaler TM. Dental predictors of high caries increment in children. J Dent Res. 1992;71:1926-33.

6. Russell JI, MacFarlane TW, Atchison TC, Stephen KW, Burchell CK. Prediction of caries increment in Scottish adolescent. Community Dent Oral Epidemiol. 1991;19:74-7.

7. Demers M, Broader JM, Mouton C, Simard PL, Trahan L, Veilleux G. A multivariate model to predict caries increment in Montreal children aged 5 years. Community Dent Health. 1992;9:273-81.

8. Van Houte J. Microbiological predictors of caries risk. Adv Dent Res. 1993;7(2):87-96.

9. Fors $\mathrm{GH}$, Sandberg $\mathrm{CH}$. Computer - aided risk management - A software tool for the Hidep model. Quintessence Int. 2001;32:309-20.

10. University of Toronto (homepage on Internet). Siyam A, Zangooei B, Sourial M, Sokary M, Soliman M, Shetty S, et al. Predictors of new caries in children and adolescents. - An evidence based report. (cited 2013 Mar 13) Avaible from: http:// www.utoronto.ca/dentistry/newsresources/evidence_based/PredictorsNewCaries.pdf.

11. The Swedish Council on Technology Assessment in Health Care. Caries - diagnosis, risk assessment and non-invasive treatment. A systematic review. Summary and conclusions. Report No 188. Stockholm: SBU; 2007.

12. Oral Health Surveys: Basic methods. 4th ed. Geneva: WHO; 1997.

13. Hänsel Petersson G, Twetman S, Bratthall D. Evaluation of a computer program for caries risk assessment in schoolchildren. Caries Res. 2002;36:327-40.

14. Moss EM. Efficacy not clearly established for professional caries prevention methods in high - risk patients. J Evid Base Dent Pract. 2002;2:199-201.

15. Bader JD, Perrin NA, Maupomé G, Rindal BD, Rush W. Validation of a simple approach to caries risk assessment. J Public Health Dent. 2005;25(2):76-81.

16. Samardi R, Gabre P, Gahnberg L. Strategies for caries risk assessment in children and adolescent at public dental clinics in a Swedish country. Int J Paediatr Dent. 2009;19(2):135-40.

17. Hänsel-Petersson G, Fure S, Bratthall D. Evaluation of a computer based caries risk assessment program in an elderly group of individuals. Acta Odont Scand. 2003;61(3):164-71.
18. Hänsel-Petersson G, Isberg PE, Twetman S. Caries risk profiles in schoolchildren over 2 years assessed by Cariogram. Int J Paediatr Dent. 2010;20(5):341-6.

19. Bratthall D, Hänsel Petersson G. Cariogram - a multifactorial risk assessment model for a multifactorial disease. Community Dent Oral Epidemiol. 2005;33:256-64.

20. Holgerson PL, Twetman S, Stecksèn-Blicks C. Validation of an age-modified caries risk assessment program (Cariogram) in preschool children. Acta Odontol Scand. 2009;67(2):106-12.

21. Utreja D, Simratvir M, Kaur A, Kwatra KS, Singh P, Dua V. An evaluation of the Cariogram as a predictor model. Int Dent J. 2010;60(4):282-4.

22. Hänsel Petersson G. Assessing caries risk - using the Cariogram model. Swed Dent J Suppl. 2003;(158):16.

23. Page RC, Martin A, Loeb CF. The Oral health Information Suite (OHIS): Its use in the management of periodontal disease. J Dent Educ. 2005;69(5):509-20.

24. Page RC, Martin J, Krall EA, Mancl L, Garcia R. Longitudinal validation of a risk calculator for periodontal disease. J Clin Periodontol. 2003;30:819-27.

25. Persson Rutger G, Mancl L, Martin J, Page RC. Assessing periodontal disease risk - a comparison of clinicians assessment versus a computerized tool. J Am Dent Assoc. 2003;134(5):575-82.

26. Page RC, Krall EA, Martin J, Mancl L, Garcia R. Validity and accuracy of a risk calculator in predicting periodontal disease. J Am Dent Assoc. 2002;133(5):569-76.

27. American Academy of Pediatric Dentistry (homepage on the Internet). Policy on use of a Caries risk Assessment Tool (CAT) for infants, children and adolescents. Oral Health Policies. (cited 2013 Mar 13) Avaible from: http://www. aapd.org/members/referencemanual/pdfs/0203/P_CariesRiskAssess.pdf.

28. Horowitz A. A Report on the NIH Consensus Development Conference on Diagnosis and Management of Dental Caries Throughout Life. J Dent Res. 2004;83(Spec Iss C):C15-C17.

29. Zero D, Fontana M, Lennon M, Dent B. Clinical applications and outcomes of using indicators of risk in caries management. J Dent Educ. 2001;65(10):1126-32.

30. Powell VL. Caries risk assessment: relevance to the practitioner. JADA. 1998;129:349-53.

31. Zukanović A, Kobašlija S, Ganibegović M. Caries risk assessment in Bosnian children using cariogram computer model. Int Dent J. 2007;57(3):177-83. 